\title{
NEW STRIP DRAWING EXPERIMENTS USING TRANSPARENT SAPPHIRE DIES
}

\author{
R. S. RAO \\ Department of Mechanical Engineering, University of Michigan, Ann Arbor, MI 48109, U.S.A. \\ M. L. DevenPECK
}

Department of Mechanical \& Industrial Engineering, Clarkson University, Potsdam, NY 13676, U.S.A.

P. K. WRIGHT

Department of Mechanical Engineering, Carnegie-Mellon University, Pittsburgh, PA 15213, U.S.A.

E. J. APPLEBY

Director of Information Services, St. Vincent College, Latrobe, PA 15650, U.S.A.

C. Y. Lu

Department of Mechanical Engineering, University of Illinois, Urbana, IL 61801, U.S.A.

and

O. RICHMOND

ALCOA Technical Center, Pittsburgh, PA 15069, U.S.A.

(Received 21 December 1984; in revised form 20 July 1985)

\begin{abstract}
Experimental results are presented for carefully controlled plane-strain strip drawing experiments. Transparent aluminum oxide (sapphire) dies have been used to make direct observations of the die-work interface in strip drawing, including the distortion of scribed lines which were used for estimating velocity profiles at the interface. Such profiles can be used as input to theoretical models (Appleby et al., 1984), thus permitting the calculation of interface friction rather than requiring its assumption. Experiments were conducted with tungsten carbide, as well as sapphire, dies in which process parameters such as reduction, speed, die angle, lubricant, and back tension were varied, and die separating forces and drawing loads were measured. In general the friction was lower for the sapphire dies, but the variation with process parameters was similar. For one experiment, residual stresses in the product strip were measured. The results are believed to be documented in sufficient detail to be of general use in evaluating theoretical models.
\end{abstract}

\section{INTRODUCTION}

Theoretical methods have found increasing application in metal forming analyses [2]. Computer programs have been developed to address problems involving large plastic strains; the goals are to predict working forces, local stress distributions in the deformation zone, and residual stress and strain distributions in the formed product. Experimental data for the quantitative evaluation of these programs have, however, been so far lacking. The strip drawing experiments recorded here provide the opportunity to evaluate many of the forming parameters, under carefully controlled plane-strain conditions. An important aim of the work has been to gather a reference collection of experimental data covering parameters such as die angle, reduction, speed, back tension, and interface friction variations. Two die materials and two lubricants have been used. Then, elsewhere [1], these experimental data have been compared with the results from FIPDEF, a finite element elasto-plastic code.

Quantitative predictions with process models depend on a knowledge of the properties of the material being worked and on the exact specification of boundary conditions. This paper emphasizes the boundary conditions in particular. A novel aspect of the experiments has been the use of transparent aluminum oxide (sapphire) dies in order that interface displacements be observed directly and relative velocities determined. The opportunity to directly observe and photograph the interface has also provided additional information on tribological features between strip and die. The principal intent of the velocity measurements, however, was to provide boundary condition information at the tool interface which could be used directly in models in place of assumed coefficients of friction. Residual stress measurements have also been carried out in some detail for one experiment, and a large number of 
experiments have been carried out to show the comparative behavior of sapphire dies with tungsten carbide dies under various process conditions.

\section{EXPERIMENTAL APPARATUS AND MATERIALS}

The experimental apparatus consisted of a pair of identical dies, either tungsten carbide or transparent sapphire, with a linear bearing between the bottom die and a load cell. In this way it was possible to monitor the die-separating force. The support for the upper die contained a viewing aperture for observing and recording the interfacial behavior when the transparent sapphire dies were used. The gap between the dies was set by means of blocks between the upper die support and the frame of the fixture. The whole apparatus was attached to a horizontal tension testing machine which had a provision to record drawing force. Figure 1 shows an overall view of the experimental set-up including a microscope with a camera.

Synthetic sapphire is aluminum oxide, grown artificially as a single crystal. It was ground and polished to die angles of $4^{\circ}$ and $8^{\circ}$. In the present work the term 'die angle' refers to the angle between one die face and the strip center line. The design of the tungsten carbide was the same as the sapphire dies, except that the carbide dies had a larger base area. The work material was tin plated mild steel, $51 \mathrm{~mm}$ wide and $0.3 \mathrm{~mm}$ thick. The electrolytically applied tin coating on each surface of the strip was about $0.38 \mu \mathrm{m}$ thick. Light mineral oil and emulsified mineral oil $(5 \%$ mineral oil by volume in water) were chosen as lubricants.

The tin plated mild steel was tested in tension at a nominal strain rate of about $0.1 \mathrm{~s}^{-1}$ to find its plastic tensile behavior. The experimental stress-strain data, obtained by uniform straining up to about $15 \%$ was fitted by the following equations:

and

$$
\sigma=m \varepsilon+b \text { for } 0 \leqslant \varepsilon \leqslant 0.028(m=1900 \mathrm{MPa}, \mathrm{b}=269 \mathrm{MPa})
$$

$$
\sigma=K \varepsilon^{n} \text { for } \varepsilon>0.028(K=570 \mathrm{MPa}, n=0.16) \text {. }
$$

In these equations, $\sigma$ and $\varepsilon$ are uniaxial true stress and true plastic strain, respectively.

\section{EXPERIMENTAL PROCEDURES AND RESULTS}

\section{Velocity measurements}

For the purpose of quantifying velocities along the interface, lines were scribed on the strip surface by means of a Rockwell scratch micro-hardness tester with a $50 \mathrm{~g}$ load. In order to magnify the image of the interface, photographs were taken through a Wild microscope with adapter tube on a $35 \mathrm{~mm}$ still camera with motor drive. Fiber optic light pipes were used for illumination. The microscope magnification setting was $25 \times$. Drawing experiments were carried out at the slow speed of $0.085 \mathrm{~mm} \mathrm{~s}^{-1}$. Filming was carried out using $35 \mathrm{~mm}$ black-and-white film at a speed of 3.2 frames $s^{-1}$. The overall magnification of the interface photographic prints was about $80 \times$.

Two different patterns of scribed lines were used in obtaining the velocities along the interface. In the first case, lines were scribed perpendicular to the direction of motion and parallel to each other with $0.25 \mathrm{~mm}$ spacing. The necessity for an accurately measured time interval was eliminated by measuring the displacement of a grid line which had exited the die as well as those within the die contact. The detailed procedure for determining the velocities from such a pattern is given in reference [3]. In the present paper a new technique using inclined lines is presented. This technique can provide more continuous information than the above mentioned technique.

A photograph of the inclined lines taken through a sapphire die is shown in Fig. 2. As the strip enters the die region the lines become curved and as the strip exits from the die region the lines appear straight again. The slope of the lines at any position can be related directly to the corresponding velocity ratio through the equation below:

$$
\frac{V_{i}}{V_{n}}=\frac{m_{n}}{m_{i}}
$$

where $V_{1}$ and $m_{i}$ are the velocity and slope at any position within the die zone and $V_{n}$ and $m_{n}$ are velocity and slope at the exit. Thus in addition to the reference values, $m_{n}$ and $V_{n}, m_{i}$ must be measured at each place within the die zone where an interfacial velocity is required. However, the changes in the angle are very small and not easy to measure accurately.

In the present work the following procedure was used. One convenient line was chosen from each of 31 photographs, similar to Fig. 2 . The coordinates of several points on each curve were obtained using a digitizer. The points on each curve were then fitted to a cubic equation. The slope at any position within the die region was obtained by differentiating the fitted cubic equation and substituting the corresponding value of the die position. Then the relative velocity was calculated using equation (1). An average velocity profile was then obtained for the 31 lines with the result shown in Fig. 3. The shaded area represents the bounds of the variations in the 31 individuals lines. The experimental scatter is most obvious at the entry and exit areas.

\section{Interfacial observations}

Photographs, taken through transparent dies, are shown in Figs $4-6$ for 10, 20 and $30 \%$ thickness reductions, and a $4^{\circ}$ die angle. Light mineral oil lubricant was used in these experiments.

Measurement of die forces and global friction

An effective coefficient of friction can be calculated for strip drawing by assuming that the interface is a plane, that there is no land (bearing area at the exit) and by balancing the forces on the die [4]. That is,

$$
\mu=\frac{((F-B) / 2 S)-\tan \theta}{[1+((F-B) / 2 S) \tan \theta]}
$$




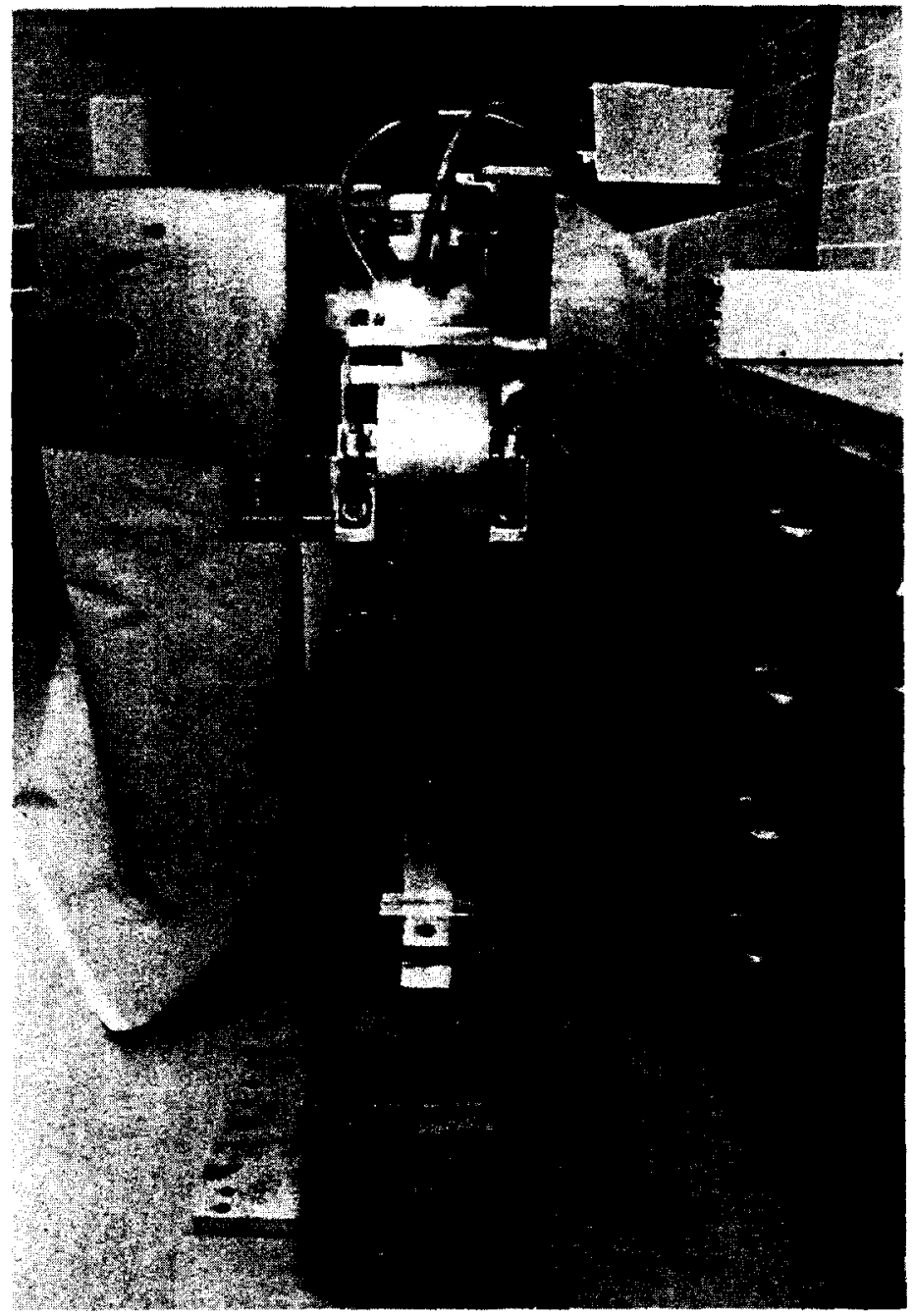

FIG. 1. Overall experimental set-up. 


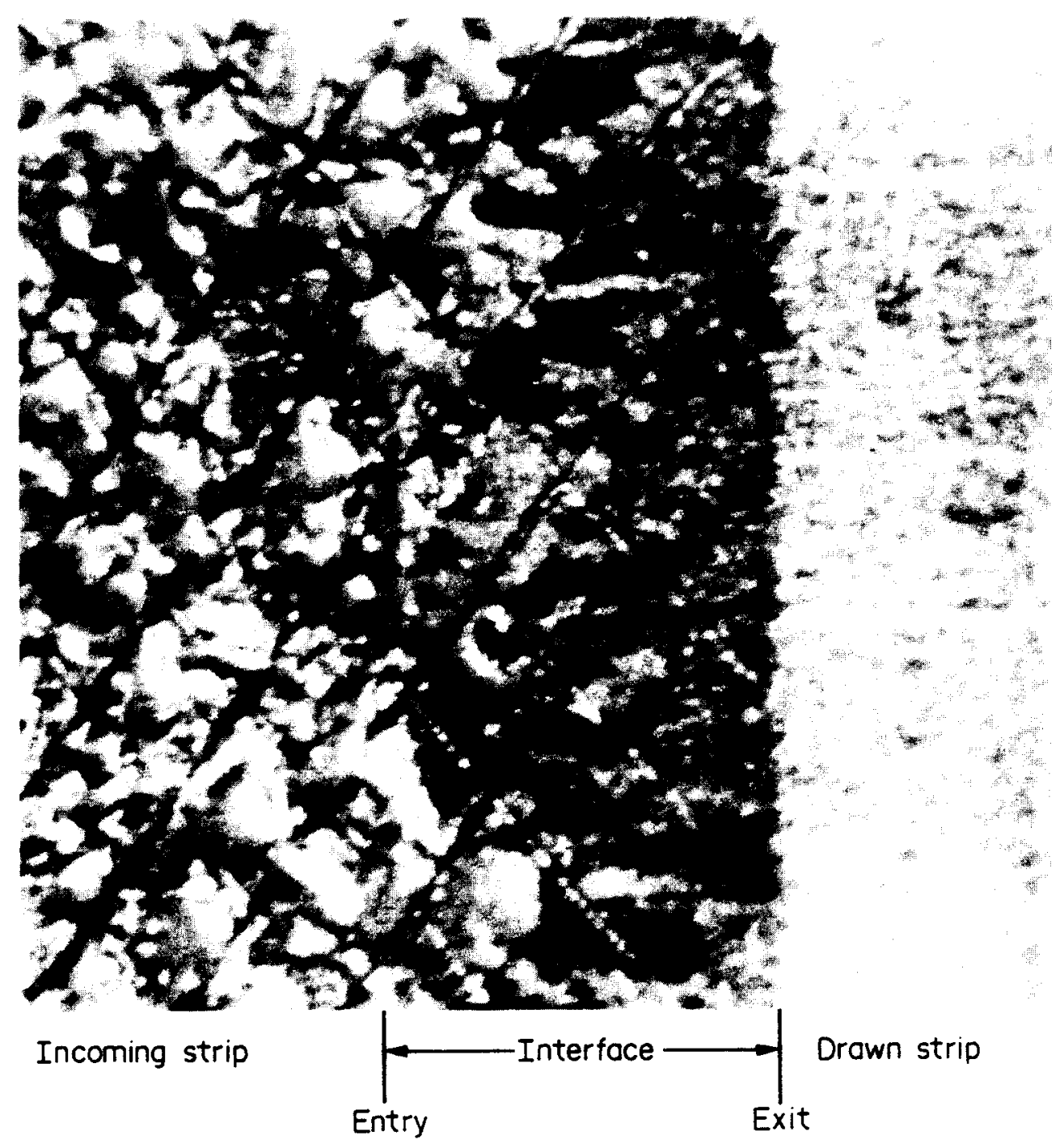

Fic. 2. A photograph of the inclined line technique. 


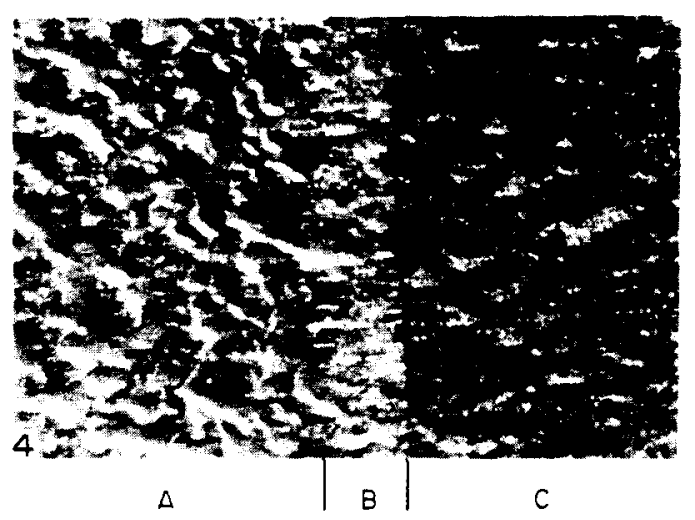

$\Delta=$ Incoming strip

$B=$ Die deformation zone

$C=$ Drown strip

Fig. 4. $10 \%$ Reduction with light mineral oil, $4^{\circ}$ die $(25 \times)$.

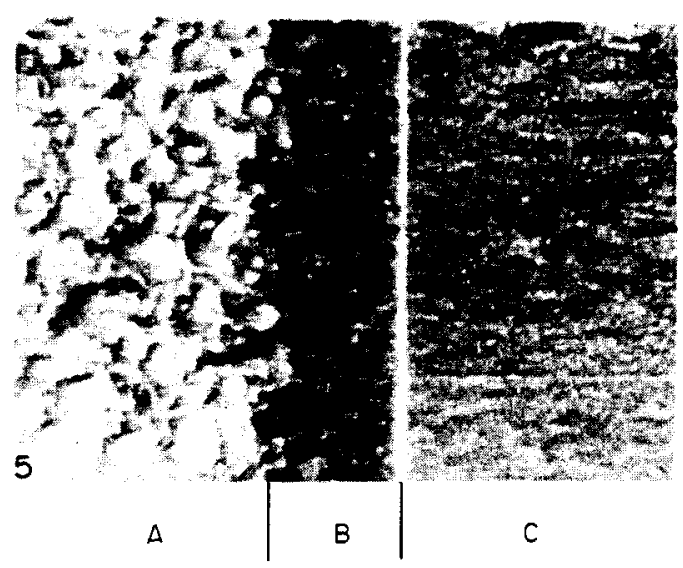

FIG. 5. $20 \%$ Reduction with light mineral oil, $4^{\circ}$ die $(25 \times)$.

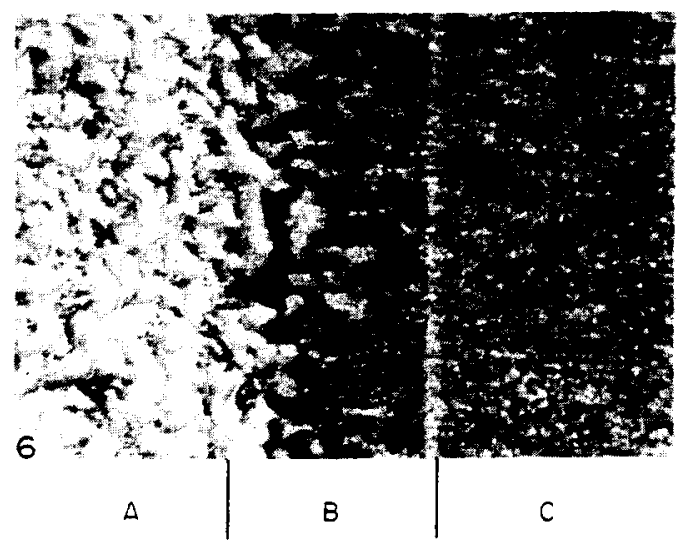

Fig. 6. $30 \%$ Reduction with light mineral oil, $4^{\circ}$ die $(25 \times)$. 



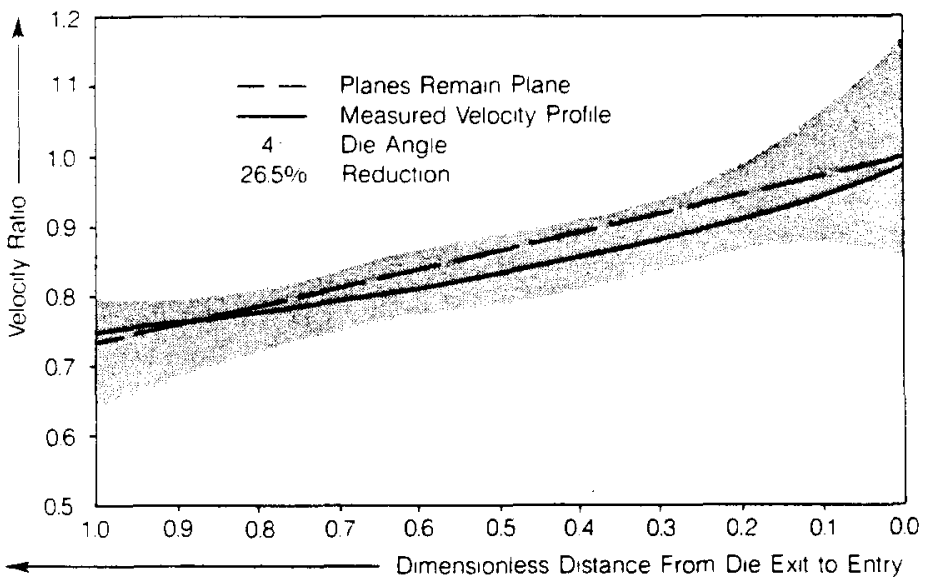

FIG. 3. The average measured velocity profile along the interface with the shaded area representing the experimental scatter.

where:

$\mu$ is effective coefficient of friction

$F$ is forward tension

$B$ is backward tension

$S$ is die-separating force

$\theta$ is die angle.

Experiments were conducted by systematically varying the following six process parameters, and the effective coefficient of friction in each case was calculated by using the appropriate experimental values in equation (3).

1. Die material: tungsten carbide and sapphire.

2. Die angle in degrees: 4 and 8 .

3. Drawing speed in $\mathrm{mm} \mathrm{s}^{-1}: 0.085,0.85$ and 8.5 .

4. Percentage reduction in thickness: 10,20 and 30.

5. Lubricant: light mineral oil and emulsified mineral oil.

6. Back tension in $N: 0,445,890$ and 1335 .

The results are presented in Tables 1-12 and the effects of these process parameters on friction are shown in Figures 7 and 8 . It should be pointed out that the percentage reduction values shown in Tables 1-12 are based on initial thickness $(0.3 \mathrm{~mm})$ and final thickness (depends on the reduction) measured using a micrometer. The tolerance on the percentage reduction is about $\pm 0.5 \%$.

Residual stress measurements

The residual stresses in a strip can be measured by appropriate removal of material. Removal of layers by chemical thinning or by a mechanical method causes imbalance of stresses and consequent changes in strain. These changes in strain due to partial relief of stress are measured and used to determine the stresses in the material removed.

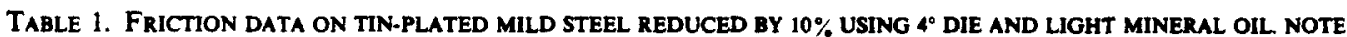
THAT IN ALL THE TABLES THE REDUCTIONS ARE $\pm 0.5 \%$ TO ACCOUNT FOR MINOR STRIP VARLATIONS AND THE ACCURACY OF THE MICROMETER

\begin{tabular}{|c|c|c|c|c|c|c|c|c|}
\hline \multirow{2}{*}{$\begin{array}{l}\text { Exp. } \\
\text { No. }\end{array}$} & \multirow{2}{*}{$\begin{array}{l}\text { Drawing } \\
\text { Speed in } \\
\mathrm{mm} / \mathrm{sec}\end{array}$} & \multirow{2}{*}{$\begin{array}{l}\text { Black } \\
\text { Tension } \\
\text { in } \mathbf{N} \\
\text { (B) }\end{array}$} & \multicolumn{2}{|c|}{$\begin{array}{c}\text { Forward Tension } \\
\text { in } \mathbf{N} \\
\text { (F) }\end{array}$} & \multicolumn{2}{|c|}{$\begin{array}{c}\text { Die Separating } \\
\text { Force in } \mathbf{N} \\
\text { (S) }\end{array}$} & \multicolumn{2}{|c|}{$\begin{array}{c}\text { Effective Coeffi- } \\
\text { cient of Friction } \\
\left(\mu_{\text {eff }}\right)\end{array}$} \\
\hline & & & Carbide & Sapphire & Carbide & Sapphire & Carbide & Sapphire \\
\hline 1 & 0.085 & 0 & 1135 & 797 & 3649 & 3649 & 0.085 & 0.040 \\
\hline 2 & 0.850 & $"$ & 1157 & 1068 & 3716 & 3961 & 0.085 & 0.064 \\
\hline 3 & 8.500 & $"$ & 1259 & 1184 & 4005 & 4139 & 0.085 & 0.072 \\
\hline 4 & 0.085 & 445 & 1660 & 1237 & 3916 & 3560 & 0.084 & 0.041 \\
\hline 5 & 0.850 & $"$ & 1704 & 1442 & 4050 & 3805 & 0.085 & 0.061 \\
\hline 6 & 8.500 & $"$ & 1758 & 1513 & 4183 & 3694 & 0.086 & 0.074 \\
\hline 7 & 0.085 & 890 & 2092 & 1633 & 3783 & 2915 & 0.088 & 0.057 \\
\hline 8 & 0.850 & $"$ & 2092 & 1780 & 3791 & 3026 & 0.088 & 0.076 \\
\hline 9 & 8.500 & $"$ & 2136 & 1825 & 3894 & 3026 & 0.089 & 0.083 \\
\hline 10 & 0.085 & 1335 & 2336 & 2047 & 3293 & 2893 & 0.081 & 0.052 \\
\hline 11 & 0.850 & $"$ & 2359 & 2114 & 3320 & 2893 & 0.083 & 0.064 \\
\hline 12 & 8.500 & $"$ & 2434 & 2225 & 3560 & 2982 & 0.084 & 0.078 \\
\hline
\end{tabular}


TABle 2. Friction Data ON TIN-PLATEd MILd STEEl REDUCED ay $10 \%$ USING $4^{\circ}$ DIE AND EMUSLSIFIEd MINERAL OIL

\begin{tabular}{|c|c|c|c|c|c|c|c|c|}
\hline \multirow{2}{*}{$\begin{array}{l}\text { Exp. } \\
\text { No. }\end{array}$} & \multirow{2}{*}{$\begin{array}{l}\text { Drawing } \\
\text { Speed in } \\
\mathrm{mm} / \mathrm{sec}\end{array}$} & \multirow{2}{*}{$\begin{array}{l}\text { Back } \\
\text { Tension } \\
\text { in } \mathbf{N} \\
\text { (B) }\end{array}$} & \multicolumn{2}{|c|}{$\begin{array}{l}\text { Forward Tension } \\
\text { in } N \\
\text { (F) }\end{array}$} & \multicolumn{2}{|c|}{$\begin{array}{l}\text { Die Separating } \\
\text { Force in } \mathbf{N} \\
\text { (S) }\end{array}$} & \multicolumn{2}{|c|}{$\begin{array}{l}\text { Effective Coeffi- } \\
\text { cient of Friction } \\
\left(\mu_{\text {eff }}\right)\end{array}$} \\
\hline & & & Carbide & Sapphire & Carbide & Sapphire & Carbide & Sapphire \\
\hline 1 & 0.085 & 0 & 1202 & 930 & 3827 & 3872 & 0.080 & 0.050 \\
\hline 2 & 0.850 & " & 1335 & 1108 & 4094 & 3961 & 0.092 & 0.069 \\
\hline 3 & 8.500 & $"$ & 1424 & 1153 & 4183 & 3961 & 0.099 & 0.075 \\
\hline 4 & 0.085 & 445 & 1633 & 1380 & 3738 & 3827 & 0.088 & 0.065 \\
\hline 5 & 0.850 & $\infty$ & 1691 & 1482 & 3738 & 3827 & 0.095 & 0.065 \\
\hline 6 & 8.500 & $n$ & 1807 & 1629 & 4005 & 3961 & 0.099 & 0.078 \\
\hline 7 & 0.085 & 890 & 2123 & 1825 & 3560 & 3671 & 0.084 & 0.057 \\
\hline 8 & 0.850 & $"$ & 2180 & 1985 & 3827 & 3827 & 0.098 & 0.072 \\
\hline 9 & 8.500 & $n$ & 2283 & 2092 & 4094 & 3872 & 0.099 & 0.084 \\
\hline 10 & 0.085 & 1335 & 2345 & 2225 & 3204 & 3560 & 0.087 & 0.055 \\
\hline 11 & 0.850 & 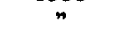 & 2492 & 2314 & 3382 & 3605 & 0.098 & 0.065 \\
\hline 12 & 8.500 & $n$ & 2590 & 2474 & 3649 & 3805 & 0.100 & 0.079 \\
\hline
\end{tabular}

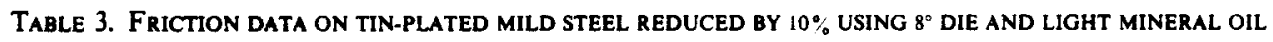

\begin{tabular}{|c|c|c|c|c|c|c|c|c|}
\hline \multirow{2}{*}{$\begin{array}{l}\text { Exp. } \\
\text { No. }\end{array}$} & \multirow{2}{*}{$\begin{array}{l}\text { Drawing } \\
\text { Speed in } \\
\mathrm{mm} / \mathrm{sec}\end{array}$} & \multirow{2}{*}{$\begin{array}{l}\text { Back } \\
\text { Tension } \\
\text { in } \mathbf{N} \\
\text { (B) }\end{array}$} & \multicolumn{2}{|c|}{$\begin{array}{l}\text { Forward Tension } \\
\text { in } \mathbf{N} \\
\text { (F) }\end{array}$} & \multicolumn{2}{|c|}{$\begin{array}{l}\text { Die Separating } \\
\text { Force in } N \\
\text { (S) }\end{array}$} & \multicolumn{2}{|c|}{$\begin{array}{l}\text { Effective Coeffi- } \\
\text { cient of Friction } \\
\left(\mu_{\text {eff }}\right)\end{array}$} \\
\hline & & & Carbide & Sapphire & Carbide & Sapphire & Carbide & Sapphire \\
\hline 1 & 0.085 & 0 & 1246 & 1010 & 2893 & 2893 & 0.073 & 0.033 \\
\hline 2 & 0.850 & " & 1304 & 1157 & 3026 & 3026 & 0.073 & 0.049 \\
\hline 3 & 8.500 & $"$ & 1424 & 1277 & 3293 & 3115 & 0.073 & 0.063 \\
\hline 4 & 0.085 & 445 & 1571 & 1424 & 2626 & 2581 & 0.072 & 0.048 \\
\hline 5 & 0.850 & $"$ & 1780 & 1558 & 3071 & 2670 & 0.075 & 0.066 \\
\hline 6 & 8.500 & $n$ & 1900 & 1660 & 3293 & 2804 & 0.078 & 0.074 \\
\hline 7 & 0.085 & 890 & 1945 & 1749 & 2448 & 2225 & 0.073 & 0.051 \\
\hline 8 & 0.850 & $"$ & 2047 & 1896 & 2581 & 2314 & 0.081 & 0.074 \\
\hline 9 & 8.500 & $"$ & 2176 & 1989 & 2804 & 2403 & 0.086 & 0.085 \\
\hline 10 & 0.085 & 1335 & 2345 & 2225 & 2314 & 2270 & 0.075 & 0.054 \\
\hline 11 & 0.850 & 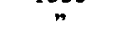 & 2492 & 2434 & 2537 & 2448 & 0.085 & 0.081 \\
\hline 12 & 8.500 & $"$ & 2600 & 2523 & 2715 & 2608 & 0.090 & 0.084 \\
\hline
\end{tabular}

TABLE 4. Friction data ON TIN-PLATEd MILD STEEl REDUCED BY $10 \%$ USING $8^{\circ}$ DIE AND EMULSIFIEd MINERAL OIL

\begin{tabular}{|c|c|c|c|c|c|c|c|c|}
\hline \multirow{2}{*}{$\begin{array}{l}\text { Exp. } \\
\text { No. }\end{array}$} & \multirow{2}{*}{$\begin{array}{c}\text { Drawing } \\
\text { Speed in } \\
\mathrm{mm} / \mathrm{sec}\end{array}$} & \multirow{2}{*}{$\begin{array}{l}\text { Back } \\
\text { Tension } \\
\text { in } N \\
\text { (B) }\end{array}$} & \multicolumn{2}{|c|}{$\begin{array}{l}\text { Forward Tension } \\
\text { in } N \\
(\mathbf{F})\end{array}$} & \multicolumn{2}{|c|}{$\begin{array}{l}\text { Die Separating } \\
\text { Force in } \mathrm{N} \\
\text { (S) }\end{array}$} & \multicolumn{2}{|c|}{$\begin{array}{l}\text { Effective Coeffi- } \\
\text { cient of Friction } \\
\left(\mu_{\mathrm{eff}}\right)\end{array}$} \\
\hline & & & Carbide & Sapphire & Carbide & Sapphire & Carbide & Sapphire \\
\hline 1 & 0.085 & 0 & 1215 & 1215 & 2759 & 2982 & 0.077 & 0.061 \\
\hline 2 & 0.850 & 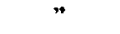 & 1304 & 1406 & 2848 & 3204 & 0.086 & 0.076 \\
\hline 3 & 8.500 & $"$ & 1380 & 1451 & 2982 & 3293 & 0.088 & 0.077 \\
\hline 4 & 0.085 & 445 & 1722 & 1513 & 2848 & 2448 & 0.081 & 0.075 \\
\hline 5 & 0.850 & " & 1825 & 1633 & 2937 & 2626 & 0.091 & 0.083 \\
\hline 6 & 8.500 & $"$ & 1958 & 1722 & 3204 & 2759 & 0.093 & 0.088 \\
\hline 7 & 0.085 & 890 & 1989 & 1900 & 2492 & 2359 & 0.078 & 0.071 \\
\hline 8 & 0.850 & $"$ & 2167 & 2000 & 2759 & 2425 & 0.088 & 0.086 \\
\hline 9 & 8.500 & $"$ & 2252 & 2136 & 2848 & 2626 & 0.095 & 0.094 \\
\hline 10 & 0.085 & 1335 & 2416 & 2345 & 2359 & 2359 & 0.086 & 0.071 \\
\hline 11 & 0.850 & $"$ & 2550 & 2492 & 2538 & 2492 & 0.096 & 0.089 \\
\hline 12 & 8.500 & $"$ & 2715 & 2600 & 2848 & 2626 & 0.098 & 0.097 \\
\hline
\end{tabular}


TABle 5. FRICTION DATA ON TIN-PLATED MILD STEEL REDUCED BY $20^{\circ}$ USING $4^{\circ}$ DIE AND LIGHT MINERAL OIL

\begin{tabular}{|c|c|c|c|c|c|c|c|c|}
\hline \multirow{2}{*}{$\begin{array}{l}\text { Exp. } \\
\text { No. }\end{array}$} & \multirow{2}{*}{$\begin{array}{l}\text { Drawing } \\
\text { Speed in } \\
\mathrm{mm} / \mathrm{sec}\end{array}$} & \multirow{2}{*}{$\begin{array}{l}\text { Back } \\
\text { Tension } \\
\text { in N } \\
\text { (B) }\end{array}$} & \multicolumn{2}{|c|}{$\begin{array}{c}\text { Forward Tension } \\
\text { in } N \\
\text { (F) }\end{array}$} & \multicolumn{2}{|c|}{$\begin{array}{l}\text { Die Separating } \\
\text { Force in N } \\
\text { (S) }\end{array}$} & \multicolumn{2}{|c|}{$\begin{array}{c}\text { Effective Coeffi- } \\
\text { cient of Friction } \\
\left(\mu_{\mathrm{efr}}\right)\end{array}$} \\
\hline & & & Carbide & Sapphire & Carbide & Sapphire & Carbide & Sapphire \\
\hline 1 & 0.085 & 0 & 2532 & 1869 & 9657 & 8188 & 0.060 & 0.044 \\
\hline 2 & 0.850 & $\ddot{*}$ & 2608 & 2016 & 9879 & 8233 & 0.060 & 0.052 \\
\hline 3 & 8.500 & $"$ & 2670 & 2163 & 10324 & 8277 & 0.059 & 0.060 \\
\hline 4 & 0.085 & 445 & 3427 & 2296 & 9345 & 8166 & 0.062 & 0.043 \\
\hline 5 & 0.850 & $"$ & 2982 & 2492 & 9612 & 8233 & 0.062 & 0.054 \\
\hline 6 & 8.500 & 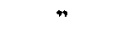 & 3071 & 2639 & 10235 & 8322 & 0.058 & 0.061 \\
\hline 7 & 0.085 & 890 & 3249 & 2639 & 8900 & 8144 & 0.061 & 0.037 \\
\hline 8 & 0.850 & $"$ & 3320 & 2759 & 9167 & 8188 & 0.062 & 0.044 \\
\hline 9 & 8.500 & $"$ & 3373 & 2937 & 9523 & 8277 & 0.060 & 0.053 \\
\hline 10 & 0.085 & 1335 & 3471 & 2968 & 8188 & 8010 & 0.062 & 0.032 \\
\hline 11 & 0.850 & $"$ & 3493 & 3115 & 8166 & 8077 & 0.062 & 0.040 \\
\hline 12 & 8.500 & $"$ & 3560 & 3262 & 8455 & 8144 & 0.061 & 0.048 \\
\hline
\end{tabular}

TABLE 6. FRICTION DATA ON TIN-PLATED MILD STEEL REDUCED BY $20 \%$ USING $4^{\circ}$ DIE AND EMULSIFIED MINERAL OIL

\begin{tabular}{|c|c|c|c|c|c|c|c|c|}
\hline \multirow{2}{*}{$\begin{array}{l}\text { Exp. } \\
\text { No. }\end{array}$} & \multirow{2}{*}{$\begin{array}{l}\text { Drawing } \\
\text { Speed in } \\
\mathrm{mm} / \mathrm{sec}\end{array}$} & \multirow{2}{*}{$\begin{array}{l}\text { Back } \\
\text { Tension } \\
\text { in } \mathbf{N} \\
\text { (B) }\end{array}$} & \multicolumn{2}{|c|}{$\begin{array}{l}\text { Forward Tension } \\
\text { in } \mathbf{N} \\
\text { (F) }\end{array}$} & \multicolumn{2}{|c|}{$\begin{array}{l}\text { Die Separating } \\
\text { Force in } N \\
\text { (S) }\end{array}$} & \multicolumn{2}{|c|}{$\begin{array}{c}\text { Efficient Coeffi- } \\
\text { cient of Friction } \\
\left(\mu_{\text {eff }}\right)\end{array}$} \\
\hline & & & Carbide & Sapphire & Carbide & Sapphire & Carbide & Sapphire \\
\hline 1 & 0.085 & 0 & 2434 & 1780 & 8900 & 8099 & 0.066 & 0.040 \\
\hline 2 & 0.850 & $"$ & 2581 & 2034 & 9078 & 8144 & 0.071 & 0.054 \\
\hline 3 & 8.500 & n & 2670 & 2194 & 9345 & 8166 & 0.072 & 0.064 \\
\hline 4 & 0.085 & 445 & 2746 & 2256 & 8544 & 8144 & 0.064 & 0.041 \\
\hline 5 & 0.850 & $"$ & 2848 & 2488 & 8633 & 8188 & 0.068 & 0.054 \\
\hline 6 & 8.500 & $"$ & 2995 & 2670 & 9078 & 8233 & 0.070 & 0.064 \\
\hline 7 & 0.085 & 890 & 3231 & 2550 & 8589 & 7943 & 0.065 & 0.034 \\
\hline 8 & 0.850 & $n$ & 3373 & 2759 & 8989 & 8032 & 0.067 & 0.046 \\
\hline 9 & 8.500 & $"$ & 3516 & 2901 & 9345 & 8077 & 0.070 & 0.054 \\
\hline 10 & 0.085 & 1335 & 3605 & 3071 & 8384 & 8010 & 0.065 & 0.038 \\
\hline 11 & 0.850 & $"$ & 3738 & 3262 & 8722 & 8055 & 0.067 & 0.049 \\
\hline 12 & 8.500 & $"$ & 3859 & 3440 & 9123 & 8099 & 0.068 & 0.059 \\
\hline
\end{tabular}

TABLE 7. FRICTION DATA ON TIN-PLATED MILD STEEL REDUCED BY $20 \%$ USING $8^{\circ}$ DIE AND LIGHT MINERAL OIL

\begin{tabular}{|c|c|c|c|c|c|c|c|c|}
\hline \multirow{2}{*}{$\begin{array}{l}\text { Exp. } \\
\text { No. }\end{array}$} & \multirow{2}{*}{$\begin{array}{l}\text { Drawing } \\
\text { Speed in } \\
\mathrm{mm} / \mathrm{sec}\end{array}$} & \multirow{2}{*}{$\begin{array}{l}\text { Back } \\
\text { Tension } \\
\text { in } \mathbf{N} \\
\text { (B) }\end{array}$} & \multicolumn{2}{|c|}{$\begin{array}{l}\text { Forward Tension } \\
\text { in } N \\
\text { (F) }\end{array}$} & \multicolumn{2}{|c|}{$\begin{array}{l}\text { Die Separating } \\
\text { Force in } \mathbf{N} \\
\text { (S) }\end{array}$} & \multicolumn{2}{|c|}{$\begin{array}{l}\text { Effective Coeffi- } \\
\text { cient of Friction } \\
\left(\mu_{\mathrm{eff}}\right)\end{array}$} \\
\hline & & & Carbide & Sapphire & Carbide & Sapphire & Carbide & Sapphire \\
\hline 1 & 0.085 & 0 & 2194 & 1691 & 5385 & 4739 & 0.061 & 0.037 \\
\hline 2 & 0.850 & $n$ & 2207 & 1869 & 5429 & 4806 & 0.061 & 0.052 \\
\hline 3 & 8.500 & $n$ & 2359 & 2047 & 5785 & 4984 & 0.062 & 0.063 \\
\hline 4 & 0.085 & 445 & 2581 & 2078 & 5207 & 4628 & 0.063 & 0.035 \\
\hline 5 & 0.850 & $"$ & 2594 & 2341 & 5251 & 4984 & 0.062 & 0.048 \\
\hline 6 & 8.500 & $"$ & 2700 & 2492 & 5607 & 5340 & 0.059 & 0.050 \\
\hline 7 & 0.085 & 890 & 3026 & 2434 & 5207 & 4397 & 0.063 & 0.034 \\
\hline 8 & 0.850 & $"$ & 3057 & 2697 & 5300 & 4539 & 0.062 & 0.057 \\
\hline 9 & 8.500 & $"$ & 3084 & 2848 & 5474 & 4762 & 0.058 & 0.063 \\
\hline 10 & 0.085 & 1335 & 3320 & 2879 & 4717 & 4272 & 0.068 & 0.039 \\
\hline 11 & 0.850 & 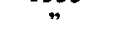 & 3320 & 3084 & 4806 & 4450 & 0.064 & 0.054 \\
\hline 12 & 8.500 & $"$ & 3382 & 3262 & 4962 & 4717 & 0.064 & 0.062 \\
\hline
\end{tabular}


TABLE 8. FRICTION DATA ON TIN-PLATED MILD STEEL REDUCED BY $20 \%$ USING $8^{\circ}$ DIE AND EMULSIFIED MINERAL OIL

\begin{tabular}{|c|c|c|c|c|c|c|c|c|}
\hline \multirow{2}{*}{$\begin{array}{l}\text { Exp. } \\
\text { No. }\end{array}$} & \multirow{2}{*}{$\begin{array}{l}\text { Drawing } \\
\text { Speed in } \\
\mathrm{mm} / \mathrm{sec}\end{array}$} & \multirow{2}{*}{$\begin{array}{l}\text { Back } \\
\text { Tension } \\
\text { in } N \\
\text { (B) }\end{array}$} & \multicolumn{2}{|c|}{$\begin{array}{l}\text { Forward Tension } \\
\text { in } N \\
\text { (F) }\end{array}$} & \multicolumn{2}{|c|}{$\begin{array}{l}\text { Die Separating } \\
\text { Force in } N \\
\text { (S) }\end{array}$} & \multicolumn{2}{|c|}{$\begin{array}{c}\text { Effective Coeffi- } \\
\text { cient of Friction } \\
\left(\mu_{\text {eff }}\right)\end{array}$} \\
\hline & & & Carbide & Sapphire & Carbide & Sapphire & Carbide & Sapphire \\
\hline 1 & 0.085 & 0 & 2314 & 1869 & 5629 & 5006 & 0.063 & 0.045 \\
\hline 2 & 0.850 & $"$ & 2448 & 1989 & 5741 & 5073 & 0.071 & 0.054 \\
\hline 3 & 8.500 & $"$ & 2581 & 2181 & 5874 & 5251 & 0.077 & 0.065 \\
\hline 4 & 0.085 & 445 & 2612 & 2181 & 5251 & 4717 & 0.064 & 0.042 \\
\hline 5 & 0.850 & $"$ & 2790 & 2403 & 5429 & 5073 & 0.073 & 0.051 \\
\hline 6 & 8.500 & $"$ & 2968 & 2612 & 5608 & 5340 & 0.082 & 0.061 \\
\hline 7 & 0.085 & 890 & 2861 & 2461 & 4762 & 4139 & 0.065 & 0.048 \\
\hline 8 & 0.850 & $*$ & 2995 & 2701 & 4940 & 4406 & 0.073 & 0.063 \\
\hline 9 & 8.500 & $"$ & 3191 & 2861 & 5162 & 4628 & 0.080 & 0.070 \\
\hline 10 & 0.085 & 1335 & 3293 & 2835 & 4717 & 3961 & 0.065 & 0.048 \\
\hline 11 & 0.850 & $"$ & 3502 & 3071 & 5029 & 4288 & 0.073 & 0.063 \\
\hline 12 & 8.500 & " & 3680 & 3204 & 5340 & 4406 & 0.077 & 0.070 \\
\hline
\end{tabular}

TABle 9. Friction DATA ON TIN-PLATED MILD STEEL REDUCED BY $30 \%$ USING $4^{\circ}$ DIE AND LIGHT MiNERAL OIL

\begin{tabular}{|c|c|c|c|c|c|c|c|c|}
\hline \multirow{2}{*}{$\begin{array}{l}\text { Exp. } \\
\text { No. }\end{array}$} & \multirow{2}{*}{$\begin{array}{l}\text { Drawing } \\
\text { Speed in } \\
\mathrm{mm} / \mathrm{sec}\end{array}$} & \multirow{2}{*}{$\begin{array}{l}\text { Back } \\
\text { Tension } \\
\text { in } \mathbf{N} \\
\text { (B) }\end{array}$} & \multicolumn{2}{|c|}{$\begin{array}{l}\text { Forward Tension } \\
\text { in } \mathbf{N} \\
\text { (F) }\end{array}$} & \multicolumn{2}{|c|}{$\begin{array}{l}\text { Die Separating } \\
\text { Force in } \mathbf{N} \\
\text { (S) }\end{array}$} & \multicolumn{2}{|c|}{$\begin{array}{c}\text { Effective Coeffi- } \\
\text { cient of Friction } \\
\left(\mu_{\text {eff }}\right)\end{array}$} \\
\hline & & & Carbide & Sapphire & Carbide & Sapphire & Carbide & Sapphire \\
\hline 1 & 0.085 & 0 & 2982 & 2194 & 10947 & 9145 & 0.065 & 0.050 \\
\hline 2 & 0.850 & 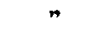 & 3026 & 2577 & 11125 & 9212 & 0.065 & 0.069 \\
\hline 3 & 8.500 & $=$ & 3115 & 2670 & 11748 & 9278 & 0.062 & 0.073 \\
\hline 4 & 0.085 & 445 & 3249 & 2690 & 10680 & 8945 & 0.060 & 0.054 \\
\hline 5 & 0.850 & $"$ & 3315 & 2817 & 10947 & 9011 & 0.061 & 0.061 \\
\hline 6 & 8.500 & n & 3338 & 2937 & 11125 & 9078 & 0.060 & 0.067 \\
\hline 7 & 0.085 & 890 & 3502 & 3146 & 9723 & 8856 & 0.064 & 0.057 \\
\hline 8 & 0.850 & $"$ & 3516 & 3235 & 9770 & 8922 & 0.064 & 0.060 \\
\hline 9 & 8.500 & $n$ & 3671 & 3382 & 10324 & 8989 & 0.064 & 0.068 \\
\hline 10 & 0.085 & 1335 & 3898 & 3404 & 9612 & 8900 & 0.063 & 0.046 \\
\hline 11 & 0.850 & $n$ & 3916 & 3636 & 9790 & 8945 & 0.062 & 0.058 \\
\hline 12 & 8.500 & $"$ & 3916 & 3769 & 10235 & 9034 & 0.056 & 0.064 \\
\hline
\end{tabular}

TABle 10. Friction DATA ON TIN-PLATED MILD STEEL REDUCED BY $30 \%$ USING $4^{\circ}$ DIE AND EMULSIFIED MINERAL OIL

\begin{tabular}{|c|c|c|c|c|c|c|c|c|}
\hline \multirow{2}{*}{$\begin{array}{l}\text { Exp. } \\
\text { No. }\end{array}$} & \multirow{2}{*}{$\begin{array}{l}\text { Drawing } \\
\text { Speed in } \\
\mathrm{mm} / \mathrm{sec}\end{array}$} & \multirow{2}{*}{$\begin{array}{l}\text { Back } \\
\text { Tension } \\
\text { in } N \\
\text { (B) }\end{array}$} & \multicolumn{2}{|c|}{$\begin{array}{c}\text { Forward Tension } \\
\text { in } N \\
\text { (F) }\end{array}$} & \multicolumn{2}{|c|}{$\begin{array}{l}\text { Die Separating } \\
\text { Force in } \mathrm{N} \\
\text { (S) }\end{array}$} & \multicolumn{2}{|c|}{$\begin{array}{c}\text { Effective Coeffi- } \\
\text { cient of Friction } \\
\left(\mu_{\text {eff }}\right)\end{array}$} \\
\hline & & & Carbide & Sapphire & Carbide & Sapphire & Carbide & Sapphire \\
\hline 1 & 0.085 & 0 & 2786 & 2372 & 10769 & 8989 & 0.059 & 0.061 \\
\hline 2 & 0.850 & $"$ & 2968 & 2550 & 11259 & 9034 & 0.061 & 0.070 \\
\hline 3 & 8.500 & $"$ & 3182 & 2640 & 11659 & 9078 & 0.066 & 0.075 \\
\hline 4 & 0.085 & 445 & 3427 & 2879 & 11036 & 9078 & 0.064 & 0.063 \\
\hline 5 & 0.850 & $"$ & 3631 & 3026 & 11570 & 9123 & 0.067 & 0.070 \\
\hline 6 & 8.500 & $"$ & 3765 & 3293 & 12015 & 9189 & 0.067 & 0.084 \\
\hline 7 & 0.085 & 890 & 3649 & 3262 & 10502 & 8989 & 0.060 & 0.061 \\
\hline 8 & 0.850 & $"$ & 3738 & 3413 & 10680 & 9078 & 0.062 & 0.068 \\
\hline 9 & 8.500 & $"$ & 3938 & 3560 & 11303 & 9167 & 0.064 & 0.075 \\
\hline 10 & 0.085 & 1335 & 3827 & 3471 & 9568 & 8811 & 0.060 & 0.051 \\
\hline 11 & 0.850 & $"$ & 3916 & 3680 & 9790 & 8900 & 0.063 & 0.060 \\
\hline 12 & 8.500 & $"$ & 4072 & 3800 & 10235 & 8945 & 0.063 & 0.067 \\
\hline
\end{tabular}


TABLE 11. FRICTION DATA ON TIN.PLATED MILD STEEL REDUCED BY $30 \%$ USING $8^{\circ}$ DIE AND LIGHT MINERAL OIL

\begin{tabular}{|c|c|c|c|c|c|c|c|c|}
\hline \multirow{2}{*}{$\begin{array}{l}\text { Exp. } \\
\text { No. }\end{array}$} & \multirow{2}{*}{$\begin{array}{l}\text { Drawing } \\
\text { Speed in } \\
\mathrm{mm} / \mathrm{sec}\end{array}$} & \multirow{2}{*}{$\begin{array}{l}\text { Back } \\
\text { Tension } \\
\text { in } N \\
\text { (B) }\end{array}$} & \multicolumn{2}{|c|}{$\begin{array}{l}\text { Forward Tension } \\
\text { in } N \\
\text { (F) }\end{array}$} & \multicolumn{2}{|c|}{$\begin{array}{l}\text { Die Separating } \\
\text { Force in } N \\
\text { (S) }\end{array}$} & \multicolumn{2}{|c|}{$\begin{array}{c}\text { Effective Coeff- } \\
\text { cient of Friction } \\
\left(\mu_{\text {eff }}\right)\end{array}$} \\
\hline & & & Carbide & Sapphire & Carbide & Sapphire & Carbide & Sapphire \\
\hline 1 & 0.085 & 0 & 2964 & 2390 & 7209 & 6586 & 0.063 & 0.040 \\
\hline 2 & 0.850 & " & 2995 & 2639 & 7343 & 6809 & 0.062 & 0.052 \\
\hline 3 & 8.500 & $"$ & 3026 & 2817 & 7498 & 7031 & 0.060 & 0.058 \\
\hline 4 & 0.085 & 445 & 3249 & 2830 & 6800 & 6453 & 0.064 & 0.043 \\
\hline 5 & 0.850 & $"$ & 3280 & 3084 & 6942 & 6702 & 0.062 & 0.055 \\
\hline 6 & 8.500 & $"$ & 3369 & 3262 & 7262 & 6900 & 0.059 & 0.062 \\
\hline 7 & 0.085 & 890 & 3649 & 3217 & 6497 & 6186 & 0.070 & 0.046 \\
\hline 8 & 0.850 & $"$ & 3707 & 3418 & 6764 & 6319 & 0.066 & 0.058 \\
\hline 9 & 8.500 & $"$ & 3783 & 3560 & 7120 & 6453 & 0.061 & 0.065 \\
\hline 10 & 0.085 & 1335 & 3872 & 3529 & 6141 & 5741 & 0.064 & 0.049 \\
\hline 11 & 0.850 & $"$ & 3916 & 3725 & 6372 & 5896 & 0.060 & 0.060 \\
\hline 12 & 8.500 & $"$ & 3996 & 3860 & 6519 & 6186 & 0.062 & 0.061 \\
\hline
\end{tabular}

TABLE 12. FRICTION DATA ON TIN-PLATED MILD STEEL REDUCED BY $30 \%$ USING $8^{\circ}$ DIE AND EMULSIFIED MINERAL OIL

\begin{tabular}{|c|c|c|c|c|c|c|c|c|}
\hline \multirow{2}{*}{$\begin{array}{l}\text { Exp. } \\
\text { No. }\end{array}$} & \multirow{2}{*}{$\begin{array}{l}\text { Drawing } \\
\text { Speed in } \\
\mathrm{mm} / \mathrm{sec}\end{array}$} & \multirow{2}{*}{$\begin{array}{l}\text { Back } \\
\text { Tension } \\
\text { in } N \\
\text { (B) }\end{array}$} & \multicolumn{2}{|c|}{$\begin{array}{l}\text { Forward Tension } \\
\text { in } N \\
\text { (F) }\end{array}$} & \multicolumn{2}{|c|}{$\begin{array}{l}\text { Die Seperating } \\
\text { Force in } N \\
\text { (S) }\end{array}$} & \multicolumn{2}{|c|}{$\begin{array}{l}\text { Effective Coeffi- } \\
\text { cient of Friction } \\
\left(\mu_{\text {eff }}\right)\end{array}$} \\
\hline & & & Carbide & Sapphire & Carbide & Sapphire & Carbide & Sapphire \\
\hline 1 & 0.085 & 0 & 2848 & 2505 & 6853 & 6631 & 0.065 & 0.047 \\
\hline 2 & 0.850 & $"$ & 3084 & 2670 & 7031 & 6853 & 0.076 & 0.053 \\
\hline 3 & 8.500 & $"$ & 3204 & 2786 & 7209 & 6898 & 0.079 & 0.060 \\
\hline 4 & 0.085 & 445 & 3262 & 3186 & 6675 & 6853 & 0.068 & 0.058 \\
\hline 5 & 0.850 & $"$ & 3471 & 3382 & 6942 & 7209 & 0.075 & 0.061 \\
\hline 6 & 8.500 & $"$ & 3591 & 3560 & 7120 & 7476 & 0.078 & 0.066 \\
\hline 7 & 0.085 & 890 & 3484 & 3306 & 6141 & 6319 & 0.069 & 0.049 \\
\hline 8 & 0.850 & $"$ & 3694 & 3591 & 6453 & 6542 & 0.074 & 0.064 \\
\hline 9 & 8.500 & $"$ & 3885 & 3769 & 6675 & 6764 & 0.081 & 0.070 \\
\hline 10 & 0.085 & 1335 & 3809 & 3604 & 5741 & 5518 & 0.073 & 0.063 \\
\hline 11 & 0.850 & $"$ & 3974 & 3769 & 5963 & 5785 & 0.078 & 0.068 \\
\hline 12 & 8.500 & $"$ & 4183 & 3885 & 6364 & 5920 & 0.081 & 0.073 \\
\hline
\end{tabular}

A strip that had been reduced by $30 \%$ in thickness using a $4^{\circ}$ sapphire die, was chosen for residual stress measurements. A specimen approximately $76 \mathrm{~mm}$ long was cut from the drawn strip. It was dipped in a microstop coating, then removed to allow the coating to dry. This was repeated a few times to ensure thorough coating on both sides of the specimen. The coating was then allowed to congeal for about $1 \frac{1}{2} \mathrm{~h}$, after which it was peeled away at each end of the specimen in a $12 \mathrm{~mm} \times 51 \mathrm{~mm}$ area on both sides. The tin coating on the exposed specimen was removed using a chemical solution of 10 parts hydrochloric acid, 2 parts nitric acid and 15 parts distilled water. Then the steel was removed by dipping the specimen in a chemical solution of $200 \mathrm{ml}$ distilled water, $16 \mathrm{~g}$ oxalic acid, $10 \mathrm{ml}$ sulfuric acid, $106 \mathrm{ml}$ hydrogen peroxide, and $2 \mathrm{ml}$ hydrofluric acid. Once the exposed steel was dissolved in the chemical solution, the remaining square specimen of $51 \mathrm{~mm} \times 51 \mathrm{~mm}$ was taken from the bath and the microstop coating was peeled off. A careful specimen preparation of this type was needed to avoid any possibility of influencing the existing residual stresses in the drawn strip which might have occurred if the specimen had been cut from the drawn strip by some mechanical means such as guillotining.

A strain gauge was mounted at the center of the $51 \mathrm{~mm} \times 51 \mathrm{~mm}$ specimen according to the procedure prescribed by the gauge manufacturer. In order to complete the half-bridge, a dummy gauge was used in conjunction with the active gauge on the specimen in the drawing direction and they were connected to the strain indicator. The specimen was placed on a horizontal table, gauge side facing up. Then sufficient microstop coating was applied to the gauge side of the specimen to prevent chemical removal of material on that side. Thickness was measured at four different places and an average was taken. The strain indicator reading was set to zero. First the tin coating was stripped using a chemical solution similar to the one used in specimen preparation. Then the strain reading and the corresponding thickness were recorded. It was found that about $50 \mathrm{ml}$ of steel stripper, similar to the one used in specimen preparation, was needed to remove uniform layers of steel about $0.013 \mathrm{~mm}$ in thickness. In this way it was possible to control the process to some extent. A layer of about $0.013 \mathrm{~mm}$ thickness was removed each time, and the corresponding strain was recorded. This procedure was repeated until half the thickness of the specimen was removed. The strain and corresponding specimen thickness are given in Table 3 . The analysis of the experimental 


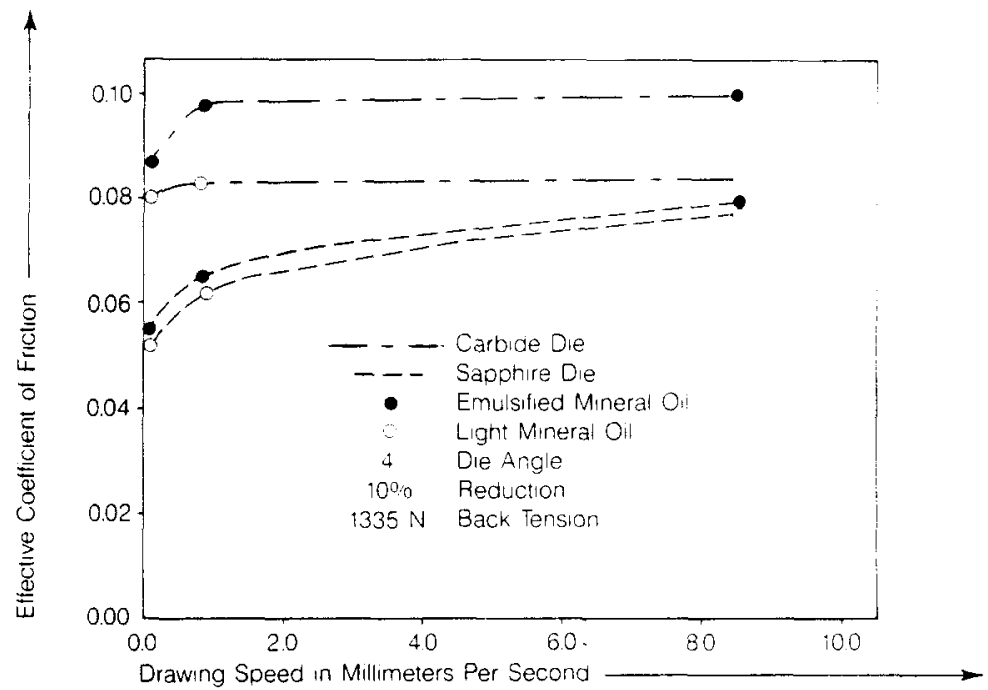

FIG. 7. A plot of drawing speed vs effective coefficient of friction.

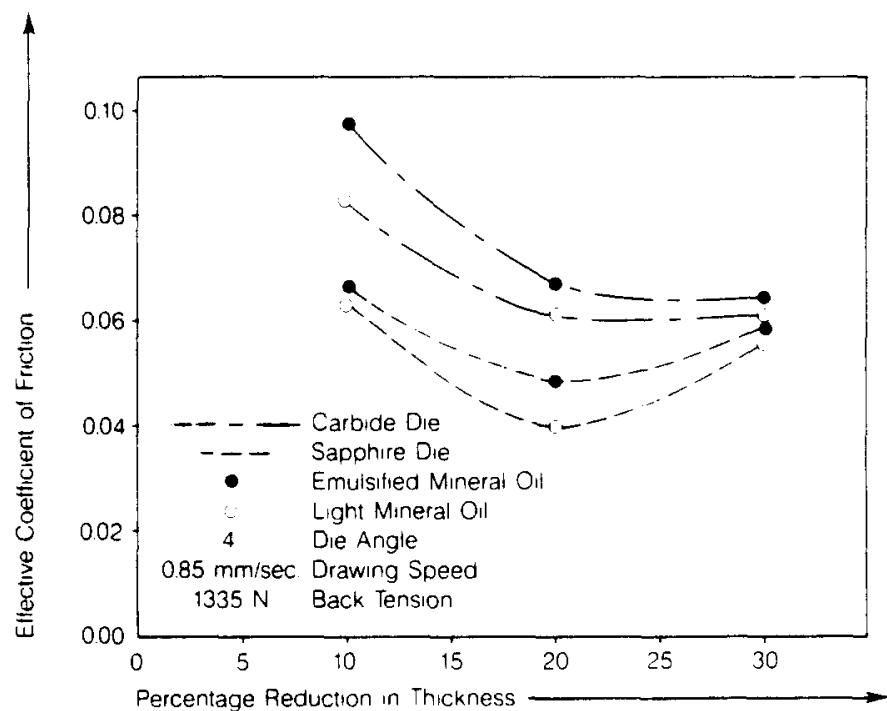

FIG. 8. A plot of percentage reduction in thickness vs effective coefficient of friction.

TABLE 13. RESIDUAL STRESS MEASUREMENTS

\begin{tabular}{|c|c|c|c|c|c|}
\hline $\begin{array}{l}\text { Exp. } \\
\text { No. }\end{array}$ & $\begin{array}{c}W \\
(\mathbf{m m})\end{array}$ & $\begin{array}{c}\mathrm{Eg} \\
\left(\times 10^{-6}\right) \\
(-)\end{array}$ & $\begin{array}{c}\mathrm{dEg} / \mathrm{dw} \\
\left(\times 10^{-3} \mathrm{~mm}^{-1}\right)\end{array}$ & $\begin{array}{c}\mathrm{Eg} / \mathrm{w}^{2} \\
\left(\times 10^{-3} \mathrm{~mm}^{-1}\right) \\
(-)\end{array}$ & $\stackrel{\sigma}{\left(\mathrm{M}^{\mathrm{Pa}}\right)}$ \\
\hline 0 & 0.2127 & 0 & 4.6520 & 0.0000 & 100 \\
\hline 1 & 0.2108 & 8 & 4.5114 & 0.1800 & 93 \\
\hline 2 & 0.2013 & 33 & 3.8154 & 0.8144 & 65 \\
\hline 3 & 0.1981 & 50 & 3.5854 & 1.2738 & 53 \\
\hline 4 & 0.1848 & 84 & 2.6327 & 2.4600 & 19 \\
\hline 5 & 0.1695 & 130 & 1.5677 & 4.5224 & -17 \\
\hline 6 & 0.1581 & 151 & 0.7862 & 6.0399 & -35 \\
\hline 7 & 0.1454 & 165 & -0.0654 & 7.8030 & -47 \\
\hline 8 & 0.1353 & 140 & -0.7335 & 7.6528 & -41 \\
\hline 9 & 0.1257 & 116 & -1.3492 & 7.3380 & -27 \\
\hline 10 & 0.1162 & 104 & -1.9552 & 7.7017 & -33 \\
\hline 11 & 0.1041 & 92 & -2.7079 & 8.4830 & -31 \\
\hline 12 & 0.0953 & 66 & -3.2524 & 7.2746 & -22 \\
\hline
\end{tabular}


results was carried out using the equation derived by Leeser and Daane [5]. That is,

$$
\sigma_{i \omega}=E\left[2 \varepsilon_{s}+\frac{1}{2} W \frac{d \varepsilon_{s}}{d W}-3 W \int_{W}^{W_{i}} \frac{\varepsilon_{\theta}}{W^{2}} \mathrm{~d} W\right]
$$

where

$\sigma_{\text {iw }}$ is residual stress when the specimen thickness is $W$

$W_{i}$ is initial specimen thickness

$\varepsilon_{g}$ is the change in strain at the gauge surface, and

$E$ is Young's modulus.

The variation of gauge strain with respect to thickness was fitted to a cubic equation and slope $\mathrm{d} \varepsilon_{\mathrm{g}} / \mathrm{d} W$ was obtained by differentiating the cubic equation. The integration part of equation (4) was carried out by the trapezoidal rule. Figure 9 presents the residual stress distribution up to the central plane of the strip.

\section{DISCUSSION}

\section{Interfacial velocities}

The measured velocity profile for a $26.5 \%$ reduction as obtained from the photographs using a $4^{\circ}$ die is shown in Fig. 3. For comparison, the linear variation in velocity along the interface from entry to exit for the special case of 'planes remain plane' is also shown. The characteristic features of the observed velocity profile are speeding up at the entry and exit and a nearly linear increase in the central region.

These empirical data have been used as the prescribed boundary conditions in a large strain elastic-plastic finite element analysis of the strip drawing process [1]. With such information, it is not necessary to assume a model of friction. Instead, the solution provides information on the local friction as well as on the stress and strain distributions, drawing load, and residual stresses.

\section{Interfacial morphology}

As shown in Figures 4-6, there are three distinct regions:

- the undeformed incoming tin plated mild steel strip,

- the die deformation zone, and

- the drawn strip.

The surface of the original tin plated strip has an undulating, or wave-like, appearance which is a result of the method by which the tin plate is produced in the temper rolling of the base

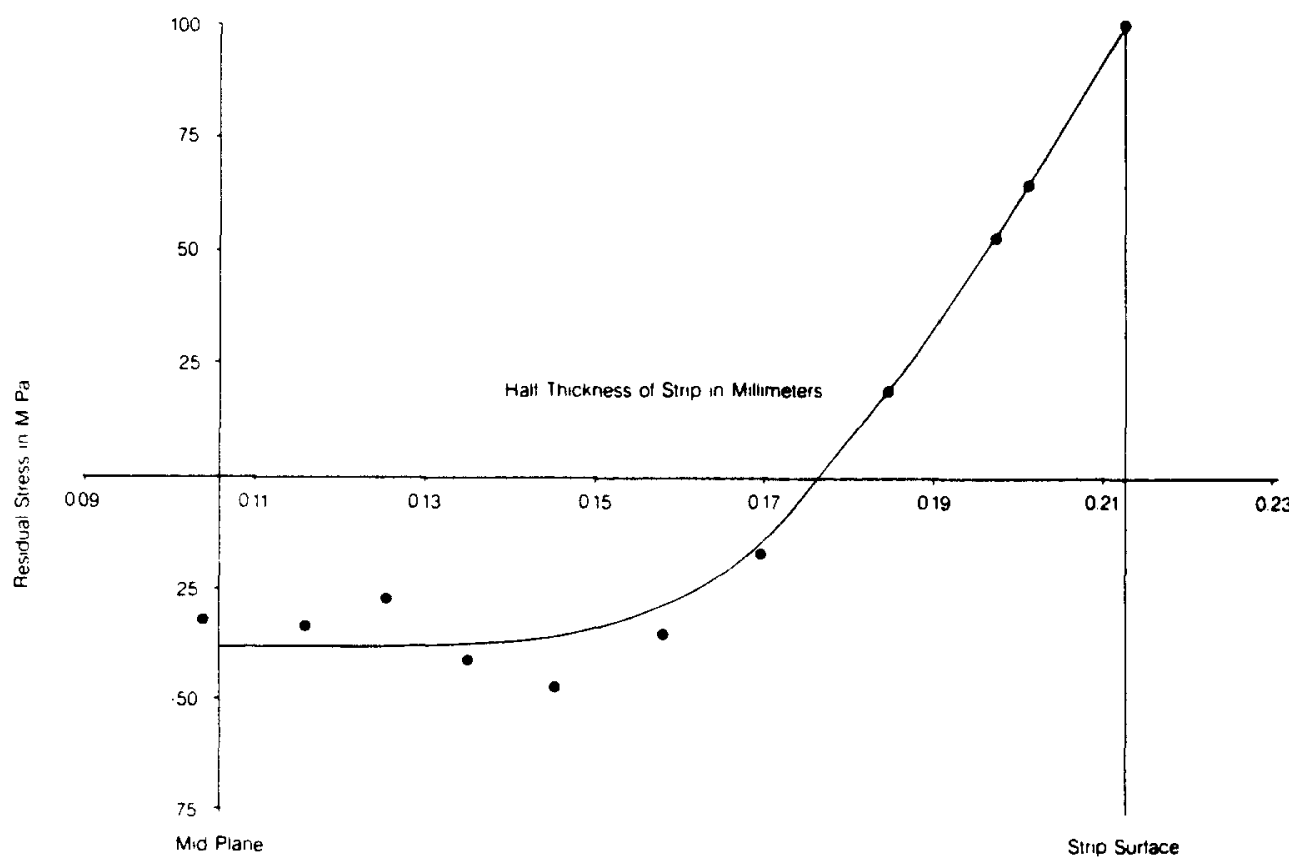

FIG. 9. Residual stress distribution. 
steel. The electrolytically applied tin represents about $0.38 \mu \mathrm{m}$ in thickness compared to the arithmetic average roughness of $1.8 \mu \mathrm{m}$. The photographs show the gradual flattening of the asperities and the resulting burnishing of the tin plate as the reduction increases through the die. During the drawing process some depressed pockets remain on the drawn strip and these continue to exhibit the matte tin finish. This is particularly evident for low reductions and low die angle, as shown in Fig. 4. As the reduction increases the pockets become smaller, as shown, for example, in Fig. 6 for $30 \%$ reduction where the entire surface is burnished. Similar features were observed for the $8^{\circ}$ die.

\section{Process parameters}

1. Die material. In general the effective coefficient of friction was lower with the sapphire dies than with the carbide dies. The ionic materials such as single crystal sapphire have a very high internal binding energy due to their 'closed structure'. Thus when placed in contact with metals such as tin plated mild steel, it is to be expected that they will not form strong bonds across the interface [6]. Another factor may be the surface roughness of the dies which is slightly less for sapphire than for carbide.

2. Die angle. It is known from the literature that there is an optimum die angle from the point of view of the combined effect of frictional and redundant work in strip drawing. In the present work the chosen die angles of $4^{\circ}$ and $8^{\circ}$ were close to the reported $[7,8]$ optimum die angle and thus the results showed only small differences in drawing loads $\left(3 \%\right.$ lower for the $4^{\circ}$ die than for the $8^{\circ}$ die for sapphire, and the reverse for carbide).

3. Drawing speed. As shown in Fig. 7, both dies showed an increase in friction with drawing speed, especially in the region below $0.7 \mathrm{~mm} \mathrm{~s}^{-1}$. Similar and opposite trends have been reported in the literature depending on the speed and lubricants used [7].

4. Reduction. It is evident from Fig. 8 that the friction generally decreased as reduction was increased. One possible explanation is that at greater reductions the surface roughness of the strip was smoothed out and the entrapped lubricant thus became a more effective film, separating the strip from the die [9].

5. Lubricant. A lubricant may reduce the friction in two ways. It serves as a reservoir for the boundary film and it prevents intermetallic contact. Figures 7 and 8 indicate that the friction was less for the light mineral oil than for the emulsified mineral oil for both types of dies.

6. Back tension. As far as the experimental results summarized in Table 1 are concerned, no regular effect of back tension on the friction was observed. The above effects can be summarized as follows:

Parameter
Speed
Reduction
Lubricant
Die material
Die angle
Back tension

Parameter

Speed

Reduction

Die material

Back tension
General influence on friction

Increasing with speed $\left(0.08-8.5 \mathrm{~mm} \mathrm{~s}^{-1}\right)$

Decreasing with reduction, especially from 10 to $20 \%$

Less for light mineral oil than for emulsified mineral oil

Less for sapphire compared to tungsten carbide

No significant difference for $4^{\circ}$ and $8^{\circ}$ observed

No regular effect

\section{Residual stress distribution}

As shown in Fig. o, the drawn strip had a residual tensile stress near the surface, of nearly $100 \mathrm{MPa}$. At $1 / 3$ of the half thickness, stress reversal occurred with a maximum compressive stress about $38 \mathrm{MPa}$ near the center. The general features of the stress distribution are reasonable and compare favorably with model predictions [1]. However they are not balanced; possibly due to some influence of the chemical-thinning technique on the stress distribution.

\section{Friction distribution along die interface}

The measured velocity profile (Fig. 3) was used as the prescribed boundary condition in the finite element analysis to provide complete information on the forces and displacements 
throughout the solution domain [1]. From this complete solution it was then of interest to analyze in detail the friction effects at the die/strip boundary. It was first necessary, for the nodes under the die, to convert the horizontal and vertical nodal forces into the die tangential and normal forces. These nodal quantities were divided into two equal parts and assigned to the two adjacent elements. The summation of the contribution from the two neighboring nodes was then assigned to the midpoint of that element side. The tangential and normal forces were plotted against the normalized die contact length as shown in the Fig. 10. Their ratio, the coefficient of friction is given in Fig. 11. The experimental scatter around the die entry and exit (see the shaded areas in Fig. 3) lead to uncertainty in the values of stress and friction coefficient in these areas. In the experimental work with the inclined lines it was especially difficult to monitor the changes in the slope of the lines at the entry. This was partly due to the very small changes in slope at the beginning of the die zone and partly due to the undulations of the tin plating which meant that the entry was a slightly spread-out zone along the strip rather than a distinct line from which measurements could be made. Examination of Fig. 2 indicates the uncertainty in determing the entry area. In fact the exit is much more distinct, hence the reason for using this as the origin in the experimental analysis. In the presentation of results (Figs 10 and 11) the uncertain areas have been shown as dashed lines. In the main body of the die zone, where the experimental scatter was small, both the friction stress and the friction coefficient rise to a peak. Physically this may be associated with the flattening of the strip and the consequent elimination of lubrication pockets as the draw

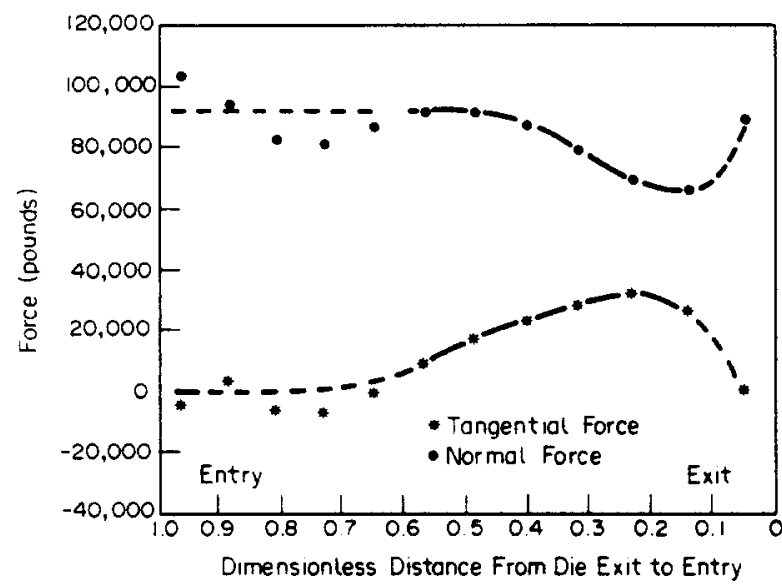

FIG. 10. Variation of normal force and tangential force along die interface using results from Fig. 3 as the boundary conditions.

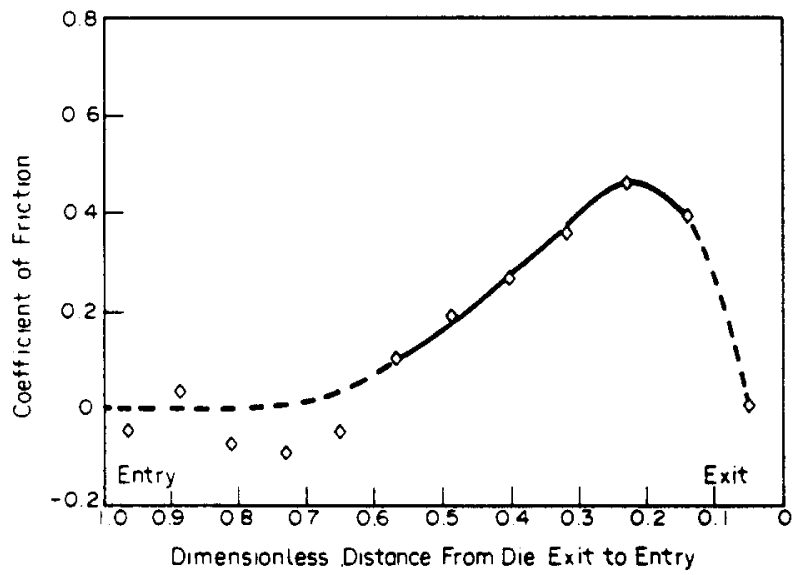

FIG. 11. Variations in the coefficient of friction. 
proceeds. The maximum value of coefficient of $\mu=0.45$ occurs approximately three-quarters of the way through the die and may be compared with a mean value of $\mu=0.06$ obtained by averaging out all the nodal values along the die (see also the effective coefficient of friction values in Table 9).

\section{CONCLUDING REMARKS}

The transparent die technique has proven to be very useful for observing the tribological conditions along the tool interface in deformation processes. By using this technique it is possible to determine the relative velocity profile at the interface which in turn can be used as input to a model of the process. In this way, it is unnecessary to assume a friction model. In fact the distribution of friction on the interface can be calculated from the model. Also, the presently constructed apparatus makes possible the observation of tribological features at the interface using movie films. The experimental results comparing sapphire with tungsten carbide dies showed less friction for sapphire, but the qualitative variations with process parameters were similar for both die materials.

Acknowledgement - This work was supported by the Solid Mechanics Division of the National Science Foundation.

\section{REFERENCES}

1. E. J. Appleby, C. Y. Lu, R. S. RAo, P. K. Wright, M. L. Devenpeck and O. Richmond, Strip drawing: a theoretical-experimental comparison. Int. J. Mech. Sci. 26, 351-362 (1984).

2. C. Y. LU, E. J. ApPLebY, R. S. RAO, M. L. DevenPECK, P. K. Wright and O. RiChmond, A numerical solution of strip drawing employing measured boundary conditions obtained with transparent sapphire. Proc. Int. Conf. on Numerical Methods in Industrial Forming Processes, Swansea, U.K., pp. $735-746$ (1982).

3. R. S. RAO, M. L. DevenPeCK, P. K. WRight, O. Richmond, E. J. Appleby and C. Y. Lu, Observations of diework interfaces through transparent sapphire dies. Proc. TMS-AIME Fall Meeting and ASM Metals Congress, St. Louis, 25-28 October, pp. 65-78 (1982).

4. A. P. Green, Plane strain theories of drawing. Proc. Inst. Mech. Engng 174, 847-864 (1960).

5. D. O. Lesser and R. A. DAANE, Residual stresses in a strip in terms of strain changes during electropolishing. Proc. Soc. Exp. Stress Anal. XII, 203-208 (1953).

6. P. K. WRIGHT and R. S. RAO, Friction reduction in machining and forming processes. North American Manufacturing Research Conference, University of Wisconsin, Madison, 24-26 May, pp. 197-202 (1983).

7. S. FuxuI, T. OHI, H. Kudo, I. TAKITA and J. SeINo, Some aspects of friction in metal-strip drawing. Int. J. Mech. Sci. 4, 297-312 (1962).

8. J. G. WISTREICH, Investigation of the mechanics of wire-drawing. Proc. Inst. Mech. Engng 169, 654-665 (1955).

9. M. L. DevenPECK and J. H. Rigo, Research apparatus for simulating high-speed drawing of thin strip. Proc. 7th North American Manufacturing Research Conf., University of Michigan, 13-16 May (1979). 